\title{
Health Plan Approach to Operationalizing a Specialty Drug Management Program
}

\author{
Mesfin Tegenu, MS, RPh
}

\begin{abstract}
BACKGROUND: Expenditures related to specialty drugs consume a significant percentage of available health care resources.

OBJECTIVE: Explain the process of transitioning the management of specialty drugs from medical services to pharmacy services in 2 managed care plans and provide insight into the issues encountered and solutions implemented based on 6 years of experience using traditional and innovative pharmacy utilization management tools to insure appropriate specialty drug use and reimbursement.

SUMMARY: The level of involvement in a specialty management program varies from managing only products dispensed by the retail, mail, and specialty pharmacy vendor to encompassing a broad list of specialty drugs distributed through a variety of channels. Efficient administrative, operational, and clinical processes are critical to the success of the program. Additionally, an accurate and timely claims processing procedure is also essential for success as is the ability to mine data and effectively report on the use of specialty products. A clinically sound, cost-effective, and patient-friendly program requires input from health plan members, pharmacy service leaders, and physician providers, and must overcome challenges associated with disrupting current relationships and removing competing incentives.
\end{abstract}

CONCLUSION: A well-constructed and properly funded specialty drug management program results in clinical and financial benefits for the plan.

J Manag Care Pharm. 2008;14(4)(suppl S):S17-S21

Copyright@ 2008, Academy of Managed Care Pharmacy. All rights reserved.

\section{Author}

MESFIN TEGENU, MS, RPh, is president of PerformRx, which is the pharmacy benefit management division of the AmeriHealth Mercy Family of Companies in Philadelphia, Pennsylvania.

AUTHOR CORRESPONDENCE: Mesfin Tegenu, MS, RPh, AmeriHealth Mercy/PerformRx, 200 Stevens Dr., 4th Fl. Pharmacy Services, Philadelphia, PA 19113. Tel.: 215.937.5013;

Fax: 215.863.5100; E-mail: mesfin.tegenu@performrx.com

\section{Introduction}

During the last 10 years, the use of specialty pharmaceutical products has increased significantly. While use of many of these products continues to be confined primarily to physician office settings, infusion clinics, or hospitals, the number of selfinjectable and oral drugs categorized as specialty drugs is growing rapidly. ${ }^{1}$ Additionally, the use of specialty products is no longer limited to rare or life threatening medical conditions. As a result of their growing number and increasing use, expenditures related to specialty drugs now consume a significant percentage of available health care resources. ${ }^{1}$

Because specialty drugs have been traditionally administered in an office or clinic setting, they were typically not covered or managed as part of the pharmacy benefit, but instead handled as part of the medical benefit. Similar to many other plans, our experience at Keystone Mercy and AmeriHealth Mercy Health plans suggests that utilization and cost management of these products was not subject to oversight by the pharmacy service department or pharmacy benefit manager. As a result, use of specialty drugs was relatively unrestricted and unmanaged, with many being administered at the discretion of the prescribing physician. Often times, these products were being utilized for unapproved uses or in situations unsupported by clinical research.

In early 2002, the senior management at the 2 health plans was charged with identifying new cost-containment opportunities. It was quickly proposed that the knowledge and expertise of the pharmacy department could be brought to bear on the acquisition, distribution, and appropriate use of specialty drug products, specifically those administered by physicians. As a result, while specialty drugs would continue to be covered under the medical benefit, the utilization and cost management of these drugs would become the responsibility of the pharmacy service area. The following is an overview of the knowledge gained from the process of transitioning management of specialty drugs from the medical benefit to the pharmacy benefit and the 6 years of experience using traditional and innovative pharmacy utilization management tools to insure appropriate use and reimbursement.

\section{Definition of Specialty Drug}

As discussions unfolded with current and potential clients of the health plans' Pharmacy Benefits Management (PBM) division, it became apparent that the definition of a specialty drug varied from client to client. For example, some clients categorized drugs as specialty if they were used to treat a specific disease state or condition, such as cancer or hemophilia. Others defined a product as specialty based on the method of administration (e.g., injectable or infusion), while still others used the location of administration (e.g., home, infusion center, physician's office, hospital, etc.) as the defining feature. There were also definitions based 
TABLE 1 Challenges Confronted When Medical Claims Data Is Used to Manage the Utilization of Specialty Drugs

- Claims data for drug use archived in multiple, segregated data systems operated by different process areas (e.g., pharmacy, PBM, medical)

- Lack of real-time access to claims data

- Data only available 30 to 90 days after date-of-administration

- Lack of usable claims data to identify provider and member utilization patterns for capitated physicians and physician groups

- Prevalent use of non-specific Healthcare Common Procedure Coding (e.g., J, S, and Q codes) to identify drug usage

- Extremely limited capability to conduct prospective, concurrent, and/or retrospective drug utilization review for specialty drug products administered and billed by physicians

- Inability to prevent duplicative payments of drugs via HCFA 1500 forms and PBM claims processing system

HCFA = Health Care Financing Administration; $P B M=$ pharmacy benefits management.

on cost tiers where agents costing more than a certain amount (e.g., > \$500, \$1000, \$1500, etc.) were defined as specialty.

\section{Coverage Concerns}

Once a product was determined by the plan to fall into the specialty drug category, the issue of coverage became the focus of discussion. The health plan had to decide if the drug in question was going to be covered as part of the member's benefit package, and if so, if it was going to be covered as part of the medical benefit or pharmacy benefit. Additionally, considerations as to who should be allowed to prescribe the drug, when, or for what condition(s) the drug should be prescribed, and specific objective requirements that must be met prior to the drug being approved for use had to be determined.

This process of identifying steps to manage the use of specialty drugs can be complicated, because these agents have historically been developed and approved for diseases and conditions that were previously untreatable or for which few satisfactory therapeutic alternatives were available. Specialty products are often the only treatments approved by the U.S. Food and Drug Administration for a given disease or condition. This reality, combined with a lack of comparative clinical trials, limited long-term outcome and economic data, and a cost factor that can be 10 to 50 times greater than existing treatment options, complicates coverage determination. Non-pharmacoeconomic factors also make coverage discussions difficult. For example, coverage of certain medications is frequently mandated by state or federal laws or insurance regulations. Direct to consumer advertising campaigns and other marketing techniques that highlight the potential benefits of the product often encourage providers, members, and advocacy groups to pressure a health plan to cover a new treatment with little regard to clinical outcomes or costeffectiveness. Once the client and the plan have categorized the specialty drug and determined coverage limitations, the existing medical or pharmacy processes are typically employed to manage utilization and distribution of the agent.

\section{Need for a Specialty Drug Management Program}

A number of issues became apparent as our pharmacy services department began to investigate the tools and/or resources that would be necessary to initiate a successful transition of specialty drug management from the medical benefit to the pharmacy benefit. The most important of these was the necessity to gain access to timely, accurate, and actionable data detailing: (1) the identity of the providers prescribing and administering specialty drugs in their office, (2) the identity of members receiving physicianadministered drugs, (3) the disease or condition the drug was being used for, (4) the strength, dosage form, and brand of specialty drug being used, (5) the amount the plan was being billed by the physician for the drug, and (6) the amount the plan was paying for the drug.

We quickly realized that incompatibilities between the medical and pharmacy information management systems made it extremely difficult to acquire data describing the use of specialty products (Table 1). In reality, the 2 systems were never designed to be cross-compatible. However, when the available medical claims data was subjected to more intense scrutiny, several trends became apparent. Among these was the fact that few, if any, financial limitations were placed on specialty drug claims submitted through the medical claims department. It also became apparent that specialty drug claims were being reviewed by staff with only a limited understanding of standard pharmacy, clinical, administrative, and reimbursement practices. As a result, oversight of hospital and outpatient surgery claims for specialty drugs carved out of Diagnosis-Related Group (DRG)-type agreements and for specialty drugs distributed through home infusion and home health care settings was limited at best.

\section{Prerequisites for Success}

As the result of experience gained during this process, our organization identified several prerequisites necessary to establish a successful specialty drug management program (Table 2). Although resolution of these issues increases the potential success of a medical benefit to pharmacy benefit transition, the degree of success depends on the ability of each health plan to reconcile the conflicting provider, patient, and distributor incentives inherent in the existing system.

\section{Program Scope}

Once the health plan decides to target specialty drugs as an opportunity to improve clinical and financial outcomes for the organization, decisions must be made regarding the level of involvement and amount of financial, human, and technology resources dedicated to implement the program. Our experience identified 5 potential targets for specialty management, including: 
(1) self-injectable and oral products, (2) office administered injectable products, (3) hospital administered products, (4) products administered in outpatient hospital and short-term stay settings, and (5) products administered via home infusion and home health care providers. The final scope of the specialty management program will vary among plans and will be greatly influenced by the quantity of resources available to fund and operate the program.

Self-injectable and oral products are typically the first and easiest group of specialty drugs to target for more aggressive management. Self-injectables and oral agents can usually be managed without dramatically changing the organizational business model or requiring large capital or staffing allocations. However, once beyond self-injectables and oral agents, management of specialty products becomes increasingly difficult. In our experience, each level of control also requires a unique set of operational, administrative, and clinical processes.

We identified a number of capabilities that must be present for any plan considering in-sourcing specialty product management. A partial list of the capabilities required for those plans interested in taking full responsibility for implementing specialty drug cost and utilization controls includes: (1) a dedicated provider and member call center, (2) claims management and processing oversight, (3) specialty drug network management, (4) rebate contracting, (5) pharmacists and technicians trained in specialty drug prior authorization protocols, and (6) clinical pharmacy specialists in specialty drug utilization management. For organizations that currently have similar responsibility and operational capacity for traditional pharmacy product management, incorporating specialty products under the existing umbrella can be a relatively easy extension of existing services. However, for those considering this as a stand-alone program, it is important to create the necessary infrastructure prior to implementation.

Unfortunately, it is very difficult to assess the human resource needs of a specialty drug management plan using algorithms associated with traditional pharmacy products and services. The resources necessary to manage specialty products are very plan specific - they depend on the number of drugs and disease types that are included in the program and the number of members within the plan requiring specialty management services. For example, the management of palivizumab, when used for the prevention of respiratory syncytial virus (RSV) in premature infants, is labor intensive and complicated by the fact that this is a seasonal program that lasts from 5 to 7 months of each year or longer if the patient resides in a region (e.g., Hawaii) that has an extended RSV season. Programs that deal with hemophilia and hepatitis $C$ are typically labor intensive early in the case management process, but require less time on a case-by-case basis over the long term.

\section{Program Strategy}

Regardless of the level of involvement the health plan ultimately chooses, our experience led us to develop a 3-phase approach to managing all specialty products. Phase 1 of the approach

\section{TABLE 2 Prerequisites for a Successful Specialty Drug Management Program}

- Health plan has identified management of specialty drugs as an opportunity to reduce overall health care costs

- The health plan has defined the scope of the specialty drug management program

- Appropriate internal and external resources have been identified and allocated to assist with the management of specialty products

- Coverage, contribution, reimbursement, distribution, and utilization management strategies for specialty drugs have been agreed to by pharmacy, medical, and management leadership

- Operational mechanism to process claims, track costs, and utilization of specialty drugs are in place

- Product and service cost issues have been identified and addressed

- Appropriate disease states, member populations, and drug products have been identified and targeted for management programs

involved reducing net cost of all drug product and services to the health plan. Phase 2 encompassed implementation of basic drug utilization management programs, and Phase 3 included the development and use of a specialty drug utilization management program. While these phases can be implemented in parallel, we found that when performed sequentially, there were fewer problems with implementation and a lower number of providerrelated issues.

Phase 1 requires that the plan closely examine how all drug products are purchased, distributed, and reimbursed. For health plans that are looking to simply control the costs and use of products currently managed under the pharmacy benefit, reviewing pharmacy purchase agreement for these products becomes critical. The plan should aggressively negotiate acquisition costs and rebates to achieve the best price available in the market for all products whether distributed by retail, mail, or specialty pharmacy networks. Also, the use of vendors who specialize in the distribution of specialty products often results in deeper discounts for those products for plans, particularly when compared with vendors who historically have focused on traditional drugs. Our experience leads us to recommend that contracts for specialty products should be drug specific; plans should avoid having a universal discount rate for all drugs covered under a single agreement. While typical retail reimbursement rates of Average Wholesale Price (AWP) minus 15\% are acceptable for some injectable products, other products are more appropriately reimbursed in the AWP-20\% range, while some brands of specialty injectable products should receive discounts up to AWP-40\%.

A common problem associated with certain specialty drug vendor agreements (e.g., hemophilia factor products) is the bundling of nursing costs or other services into the price of the drug product. These contracts often make it difficult to determine if product pricing is appropriate. Equally important, the ability to properly segregate costs and assign them to the correct internal cost center becomes extremely complicated when nursing and 
other clinical support services are bundled into the contract. To avoid these issues, we feel it is better to segregate drug product and medical/nursing services whenever possible to determine the appropriateness of the contract pricing.

The decision to expand the scope of specialty management beyond self-injectables to injectables administered in the physician office, infusion center, and home health care settings requires a different set of contracting and reimbursement strategies. In our experience, the most common type of physician reimbursement program currently in use is 'buy-and-bill.' In a buy-and-bill scheme, the physician procures the drug from a wholesaler, manufacturer, or broker. Following administration of the drug, the physician bills the health plan and/or member directly for the drug product and all drug-related professional services for reimbursement at a level frequently specified in the overall provider agreement. Our experience is that the reimbursement rates are very favorable to the physician and are often not representative of the pricing available through traditional pharmacy or specialty pharmacy vendors. It is critical that provider services contracts are reviewed and, whenever possible, modified to contain terms that are similar or identical to the reimbursement terms in the contracts with the most aggressive rate available in the retail, mail, or specialty drug vendor agreements.

In the past, it has been extremely difficult to determine the appropriate pricing of products billed by physicians due to their use of non-specific Health Care Procedure Codes (HCPC). The ability to require and use National Drug Code (NDC) level information when contracting, and reimbursing for drugs is a significant benefit. Having NDC level data, in addition to other physician and patient information, provides a more comprehensive view of physician-prescribing patterns and overall drug utilization.

Another reimbursement option is called product replacement. In a product replacement program, the physician does not bill the health plan for any drugs administered in the office, but requests a replacement item from the health plan when a drug is used for a plan member. The health plan then instructs a vendor to send a replacement product to the physician's office. The replacement cost for the drug is at the contracted rate and is often much less than the amount typically billed by the physician. This approach can be either mandatory or voluntary, however, physicians are often reluctant to sign up for this type of program without some form of incentive because participation typically means a significant reduction in profit resulting from the in-office administration and billing of specialty injectable products.

In our case, we allow buy-and-bill when the provider reimbursement contract for those products contains the same terms as those negotiated with the specialty drug replacement vendor. Additionally, we recommend the availability of a replacement drug program for those physicians who decide to use that particular process. Replacement programs offer physicians the additional advantage of reducing the time, space, and personnel requirements needed for inventory management. In many cases, the office simply requests the product from the plan immediately prior to the patient visit, thus avoiding the carrying costs associated with the more expensive specialty products.

Another issue to be aware of when contracting for specialty drug products is how to determine or negotiate contract rates. More specifically, what pricing algorithm and/or formula should be used to determine an appropriate price. AWP or Wholesale Acquisition Cost (WAC) are the most common methods of contracting with traditional pharmacy vendors. The introduction of average sales price (ASP) pricing by Centers for Medicare \& Medicaid Services (CMS) for physician reimbursement of drugs used for Medicare enrollees has added another dimension to the discussion of appropriate pricing for specialty drug products. ${ }^{2}$ Whichever baseline pricing is used, it is important to monitor pricing and modify service agreements when necessary to insure the health plan is appropriately reimbursing pharmacy and physician providers.

Phase 2 involves the development and implementation of basic drug utilization management programs. These often include point-of-service edits that screen for quantity, age, and gender, as well as prior authorization protocols that rely on objective criteria to determine if a drug is being used appropriately. The prior authorization criteria can be created internally, purchased separately from a third party, or received as a value-added component of a larger package purchased from a specialty pharmacy vendor. When appropriate, reauthorization or postauthorization review of a particular treatment is undertaken to determine effectiveness, or lack thereof, for a prescribed treatment. These basic utilization management programs and principles are the first step in helping to insure the appropriate use of specialty drug products.

Phase 3 includes the introduction of specialized pharmacy case and disease management programs. While resource intensive, these programs have been extremely cost-effective in terms of return on investment, at least from the health plan's point of view. These programs target specific diseases, conditions, or individual members that are identified based on the total cost of providing necessary care and pharmaceutical treatment. Initially, the health plans targeted medical conditions that affected a clearly identifiable group of patients who consumed a significant amount of health care resources (e.g., hemophilia, hepatitis, multiple sclerosis, end stage renal disease) for the Phase 3 specialty drug management programs.

For example, 1 of the first Phase 3 programs initiated by our pharmacy service area was for the management of hemophilia. The health plan was spending in excess of $\$ 10$ million annually on the 45 plan members identified as having the condition. The program combined the skills of a clinical pharmacist and nurse case manager to individually manage the drug therapy of these members. Use of prophylaxis factor, hospitalization from bleeding events, and the members' environment at home and school were all monitored. Shipments of drug products were also monitored and adjusted based on actual use of product as opposed to the 
standing orders for medication on record. Automatic shipment of product was discontinued, and a policy requiring all shipments to obtain authorization from the pharmacist or nurse case manager was implemented. These interventions, in concert with an aggressively renegotiated acquisition price, saved the health plan several million dollars in hospital and drug costs.

Early in the process, our plan decided to use specialty product vendors to take advantage of their expertise in the distribution of these products. We also determined that utilization management for all specialty drugs would be done by either the health plan itself or a third-party vendor that did not profit from the distribution of the drugs. While it cannot be said that all vendors would let a profit motive influence clinical judgment or decision making, this competing incentive must be considered when determining who should be given responsibility for making clinical decisions involving specialty drugs. This concern can be mitigated if the clinical criteria used to determine approval of a particular drug is provided by the health plan and not the vendor providing the product. In this case, use of a third-party vendor responsible for monitoring adherence to the prior authorization criteria would be a logical approach to reducing the chance of clinical bias.

\section{Post-Implementation Issues}

In the plan's specialty drug program, which was limited to selfinjectable and oral medications, opposition to the transition to a single or exclusive network was typically limited to physicians voicing concern about the possible disruption of relationships with current vendors or distributors. Patients have also opposed the plan and voiced their displeasure about having their preferred pharmacist or pharmacy vendor eliminated from a newly constructed specialty network. When the specialty program was expanded to include office-administered drug products, the degree of dissatisfaction often depended on the substitute distribution and reimbursement processes that were implemented. The primary complaint from physicians previously allowed to buyand-bill was predictably related to lower reimbursement rates, and the impact of this process lowered income stream to the profitability of their practice. The replacement programs also generated complaints from providers about having to front the cost of the drug to the health plan and the requirement that the physician must maintain a plan-mandated inventory, including stocking many ancillary specialty drug-related items in their offices.

Providers have also voiced concern about the additional administrative process involved with requesting replacement drug. The plan's specialty management utilization program also requires approval from the plan before the physician can even administer the drug. This has created problems, particularly when the physician requests replacement for a drug that was not authorized and, upon review, was not eligible for approval. In these cases, physicians have voiced their dissatisfaction about losing money from products that they administered, but are subsequently unable to get reimbursement.
While complaints regarding revenue and profit are certainly expected, the more consistent complaints are rooted in the requirement that providers and patients adapt to limits on the drugs that were previously unmanaged. Probably the most vocal in this regard are specialist physicians, many of whom are of the opinion that they should be exempt from any and all utilization management programs, including those established for specialty drugs. It is important for the plan to anticipate this form of resistance and devise strategies to work with their providers to resolve these issues in a mutually acceptable manner. It is our experience that plans unwilling to find workable solutions with their specialist providers will run into difficulties implementing an aggressive utilization management program.

\section{Summary}

While our experience is limited to 2 health plans, we strongly believe that a thoughtful, well-constructed, and properly funded specialty drug management program can have a positive clinical and financial impact on the plan. The level of involvement in a specialty management program can vary from touching only those products that are dispensed by the retail, mail, and specialty pharmacy vendor to encompassing a broad list of specialty drugs distributed by a variety of health care providers and vendors. Critical to the success of these programs are efficient administrative, operational, and clinical processes. The ability to duplicate an accurate and timely claims processing procedure for specialty drugs that is similar to that currently available for traditional oral drug products is essential for success. The ability to mine data and effectively report on the use of specialty products is also paramount. A clinically sound, patient-friendly, and cost-effective program involves input from health plan members, pharmacy service leaders, and physician providers.

To realize the maximum benefits of a specialty drug management program, the plan must be willing to acknowledge and overcome challenges associated with implementing a system that disrupts current relationships and removes many attractive financial incentives. Additionally, a dedicated and knowledgeable staff that understands the detailed workings of a specialty drug program and the needs of those impacted is critical. However, once the programs are in place and working, the results are well worth the effort.

\section{DISCLOSURES}

Mesfin Tegenu, MS, RPh, discloses that there was no relationship or financial interest related to the topic of this activity. Tegenu was responsible for the entire study concept and design of this article. He performed all the data collection, data interpretation, writing, and revision of this article.

\section{REFERENCES}

1. Medco Health. Drug Trend Report 2007. Available at: http://medco. mediaroom.com/file.php/129/2007+DRUG+TREND+REPORT.pdf. Accessed March 25, 2008

2. Centers for Medicare \& Medicaid Services. Medicare Part B Drug Average Sales Price. Available at: www.cms.hhs.gov/McrPartBDrugAvgSalesPrice/. Accessed March 25, 2008. 